\title{
Design of a Transportable High Efficiency Fast Neutron Spectrometer
}

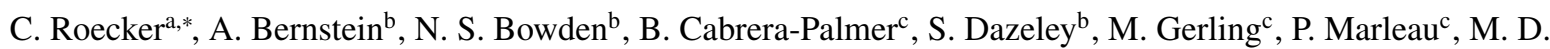 \\ Sweany $^{\mathrm{c}}, \mathrm{K}$. Vetter ${ }^{\mathrm{a}, \mathrm{d}}$ \\ ${ }^{a}$ Department of Nuclear Engineering, University of California at Berkeley, CA 94720, USA \\ ${ }^{b}$ Nuclear and Chemical Sciences Division, Lawrence Livermore National Laboratory, Livermore, CA 94550, USA \\ ${ }^{c}$ Radiation and Nuclear Detection Systems, Sandia National Laboratories, Livermore, CA 94550, USA \\ ${ }^{d}$ Nuclear Science Division, Lawrence Berkeley National Laboratory, Berkeley, CA 94720, USA
}

\section{Abstract}

A transportable fast neutron detection system has been designed and constructed for measuring neutron energy spectra and flux ranging from tens to hundreds of $\mathrm{MeV}$. The transportability of the spectrometer reduces the detectorrelated systematic bias between different neutron spectra and flux measurements, which allows for the comparison of measurements above or below ground. The spectrometer will measure neutron fluxes that are of prohibitively low intensity compared to the site-specific background rates targeted by other transportable fast neutron detection systems.

To measure low intensity high-energy neutron fluxes, a conventional capture-gating technique is used for measuring neutron energies above $20 \mathrm{MeV}$ and a novel multiplicity technique is used for measuring neutron energies above $100 \mathrm{MeV}$. The spectrometer is composed of two Gd containing plastic scintillator detectors arranged around a lead spallation target. To calibrate and characterize the position dependent response of the spectrometer, a Monte Carlo model was developed and used in conjunction with experimental data from gamma ray sources. Multiplicity event identification algorithms were developed and used with a Cf-252 neutron multiplicity source to validate the Monte Carlo model Gd concentration and secondary neutron capture efficiency. The validated Monte Carlo model was used to predict an effective area for the multiplicity and capture gating analyses. For incident neutron energies between $100 \mathrm{MeV}$ and $1000 \mathrm{MeV}$ with an isotropic angular distribution, the multiplicity analysis predicted an effective area of $500 \mathrm{~cm}^{2}$ rising to $5000 \mathrm{~cm}^{2}$. For neutron energies above $20 \mathrm{MeV}$, the capture-gating analysis predicted an effective area between $1800 \mathrm{~cm}^{2}$ and $2500 \mathrm{~cm}^{2}$. The multiplicity mode was found to be sensitive to the incident neutron angular distribution.

Keywords: fast neutron spectroscopy, neutron multiplicity, capture-gating

\section{Introduction}

The Multiplicity And Recoil Spectrometer (MARS) is a transportable fast neutron detection system for measuring neutron spectra and flux ranging from tens to hundreds of MeV. Specifically, MARS was designed to measure

*Corresponding Author Tel. +1 6202123741

Email address: calebroecker@berkeley .edu (C. Roecker) 
neutron fluxes that are of prohibitively low intensity compared to the site-specific background rates targeted by other transportable fast neutron detection systems. The transportability of the detector is a key feature which reduces the detector-related systematic bias between different neutron spectra and flux measurements.

Conventional fast neutron spectrometers for high-energy neutron fluxes utilize capture-gating [1-3]. A capturegated event sequence is as follows: an incident fast neutron undergoes multiple scatters in the detector and a single large energy deposition is recorded. In this work a deposition is defined as the integrated charge in $300 \mathrm{~ns}$ time range and an event is a collection of correlated depositions. To record the capture-gated event, the initial neutron is thermalized and captured in the detector. The capture-gated analysis infers the incident neutron spectra by unfolding the measured energy in the initial deposition using a known or simulated detector response. While capture-gating is capable of measuring high-energy neutron spectra, it may not be ideally suited for measuring high-energy neutron spectra. In particular, high-energy neutrons with energies of hundreds of $\mathrm{MeV}$ are difficult to measure due to their small macroscopic scattering cross-section and ability to escape the detector after the initial scatter.

To measure high-energy neutron fluxes MARS uses two measurement modes: a capture-gating mode for neutrons energies above $20 \mathrm{MeV}$, and a multiplicity mode for neutron energies above $100 \mathrm{MeV}$. In the multiplicity mode, a fast neutron interacts in the lead target producing multiple secondary neutrons with average energies predicted from Monte Carlo simulations ranging from $\sim 1-2 \mathrm{MeV}$ [4, 5]. These secondary neutrons thermalize and are captured producing depositions in the detector. The advantage of using the multiplicity mode is that high multiplicity events are comparatively easier to distinguish from uncorrelated neutron or gamma ray background events than in a conventional capture-gating analysis.

The first high-energy neutron flux measurement performed by MARS was at the Kimballton Underground Research Facility (KURF) near Giles, Virginia. Measurements were performed at the 380, 600, and 1450 meters water equivalent (m.w.e.) depths. Due to the transportability of MARS, the detector-related systematic bias between the three measurements was minimized, which will facilitate the creation of a robust model of the depth-dependence of the high-energy neutron flux. Predictions from this model will be used as an input to estimate backgrounds in the proposed antineutrino detector WATCHMAN [6] and future underground experiments, for which high-energy neutrons are often a dominant background. The measurement and analysis of the neutron energy spectra and flux at the three KURF depths will be presented in a future results paper. This paper focuses on the measurement concept, design, construction, calibration, and operational characteristics of MARS.

In the remainder of the paper those features of the MARS detector design, construction, and calibration that are relevant for the multiplicity mode are addressed. No spectroscopic reconstructions are presented in this work; predicted detector response changes as a function of the incident neutron energy are presented. Future papers will cover the ability of MARS to reconstruct the incident neutron energy dependent flux above and below ground. In Sec. 2 the multiplicity measurement technique used by MARS is introduced and a description of the detector is provided. In Sec. 34 the Monte Carlo model and the detector characterization by experimental and simulation methods is presented. In Sec. 5 a triggering algorithm for detecting multiplicity events is presented. In Sec. 6 the predicted 
detector response to time-correlated multiplicity events using Cf-252 neutron multiplicity source is presented. This analysis is used to validate the Monte Carlo model Gd concentration to ensure the correct secondary neutron capture efficiency. Finally in Sec. 7 the validated Monte Carlo simulation is used to predict the effective area of the MARS detector to high-energy neutrons for the multiplicity and capture-gating modes.

\section{MARS Design}

MARS was designed to be an efficient, transportable fast neutron spectrometer that uses both the multiplicity and capture-gating modes. The detection medium consists of plastic scintillator sheets interleaved with Gd coated Mylar sheets. Two such units are arranged around a lead target in which secondary neutrons are generated by the incoming fast neutrons of interest. A schematic of MARS is shown in Fig. 1

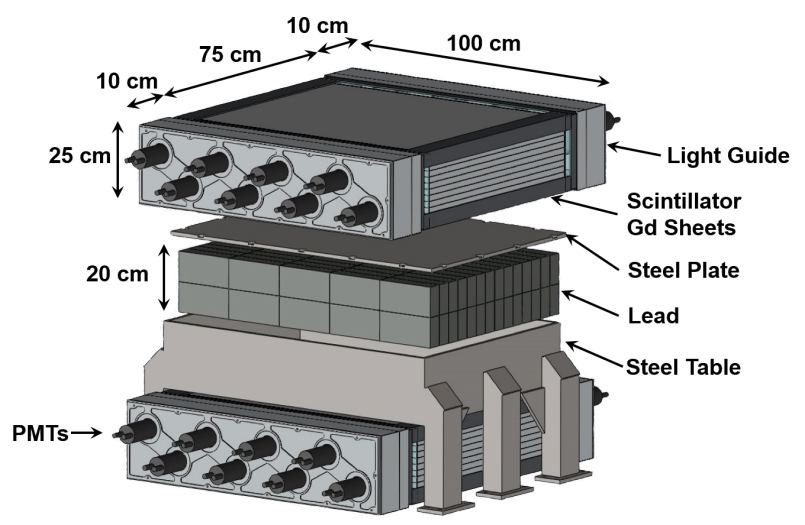

Figure 1: An exploded view illustration of the detectors, frame, and lead.

The plastic scintillator sheets provide an efficient, radiation sensitive, neutron down-scattering medium. The large thermal neutron capture cross section and energetic de-excitation of the Gd nucleus allow for identification of neutron capture interactions when coupled with the light output from the plastic scintillator. The lead is an efficient fast-toslow neutron converter. In addition to the neutron detector, MARS consists of a muon veto system and associated electronics which are housed in a mobile deployment platform. Where drive-in access is available, MARS can be transported by moving the deployment platform with a $\geq 1$-ton pickup truck. In the absence of drive-in access, the detector must be partially disassembled.

For measuring high-energy neutron spectra, MARS uses the multiplicity mode, which was previously proposed and demonstrated for high-energy neutron integrated flux measurements, but not for energy spectra measurements [7, 8]. This method has the previously unexploited potential for spectral unfolding of the primary neutron energy spectrum based on the multiplicity and other associated characteristics of the secondary neutrons. 


\subsection{Multiplicity Measurement Concept}

When a primary fast neutron interacts with the lead target as shown in Fig. 2 a, a spallation reaction can occur, resulting in multiple $\sim 1-2 \mathrm{MeV}$ secondary neutrons emerging from all sides of the target. These secondary neutrons down-scatter in the scintillator surrounding the lead and are captured by a Gd nucleus in the paint with an experimentally measured capture time of $18.7 \pm 3.0 \mu \mathrm{s}$ (Sec. 6). The excited Gd nucleus then de-excites emitting 1-5 gamma rays with a total energy of $\sim 8 \mathrm{MeV}$ which interact in the scintillator, forming a deposition. Energy depositions above $8 \mathrm{MeV}$ which are anti-coincident from the muon veto and initiate the multiplicity event trigger are ascribed to scattering from primary neutrons or secondary particle interactions not including neutron capture on Gd. Scattering or non-capture depositions are assumed to be the first deposition in a multiplicity event. Events with depositions above $8 \mathrm{MeV}$ after the first deposition are discarded. The timing structure of an example event is displayed in Fig. 2p. An event is characterized by:

1. Multiplicity: excluding the first deposition, the number of depositions with $\leq 8 \mathrm{MeV}$ deposited energy

2. Capture Energy: excluding the first deposition, the total deposited energy from all depositions

3. Thermalization Energy: the deposited energy from the first deposition

Finally, in Fig. 20 the detected secondary neutron multiplicity is displayed for several primary neutron energies spanning 100-500 MeV. The endpoint in the detected event multiplicity increased with the incident neutron energy.

\subsection{Neutron Detection System}

The neutron detection system of MARS consists of two plastic scintillator/Gd detectors with dimensions $100 \times 75 \times 25 \mathrm{~cm}^{3}$ mounted above and below a $101 \times 71 \times 20 \mathrm{~cm}^{3}$ lead converter supported by a steel table. The neutron detectors each consist of twelve $2 \mathrm{~cm}$ thick BC-408 plastic scintillator sheets interleaved with thin Mylar sheets coated with a Gd loaded paint. Each $100 \times 25 \mathrm{~cm}^{2}$ face is coupled to a $100 \times 25 \times 10 \mathrm{~cm}^{3}$ acrylic light guide. Eight 5 inch diameter ADIT B133D01 photo multiplier tubes (PMTs) [9] are coupled to the light guides by silicon grease.

The two neutron detectors are held together by a steel frame. The frame is mounted to the top and bottom of the lead containing steel table. An exploded view of the assembly of the detectors, table, and lead are shown in Fig. 1 . The table is bolted to the frame of the deployment platform.

\subsection{Muon Veto System}

The muon veto system consists of seven $72 \times 24 \times 1$ inch $^{3}$ plastic scintillator paddles coupled to fishtail light guides and PMTs on each end. These seven paddles surround the detector on the top and sides. Four more paddles with dimensions $31 \times 28 \times 2$ inch $^{3}$ cover the ends of the detector; a single PMT is mounted in the center of the paddle. 

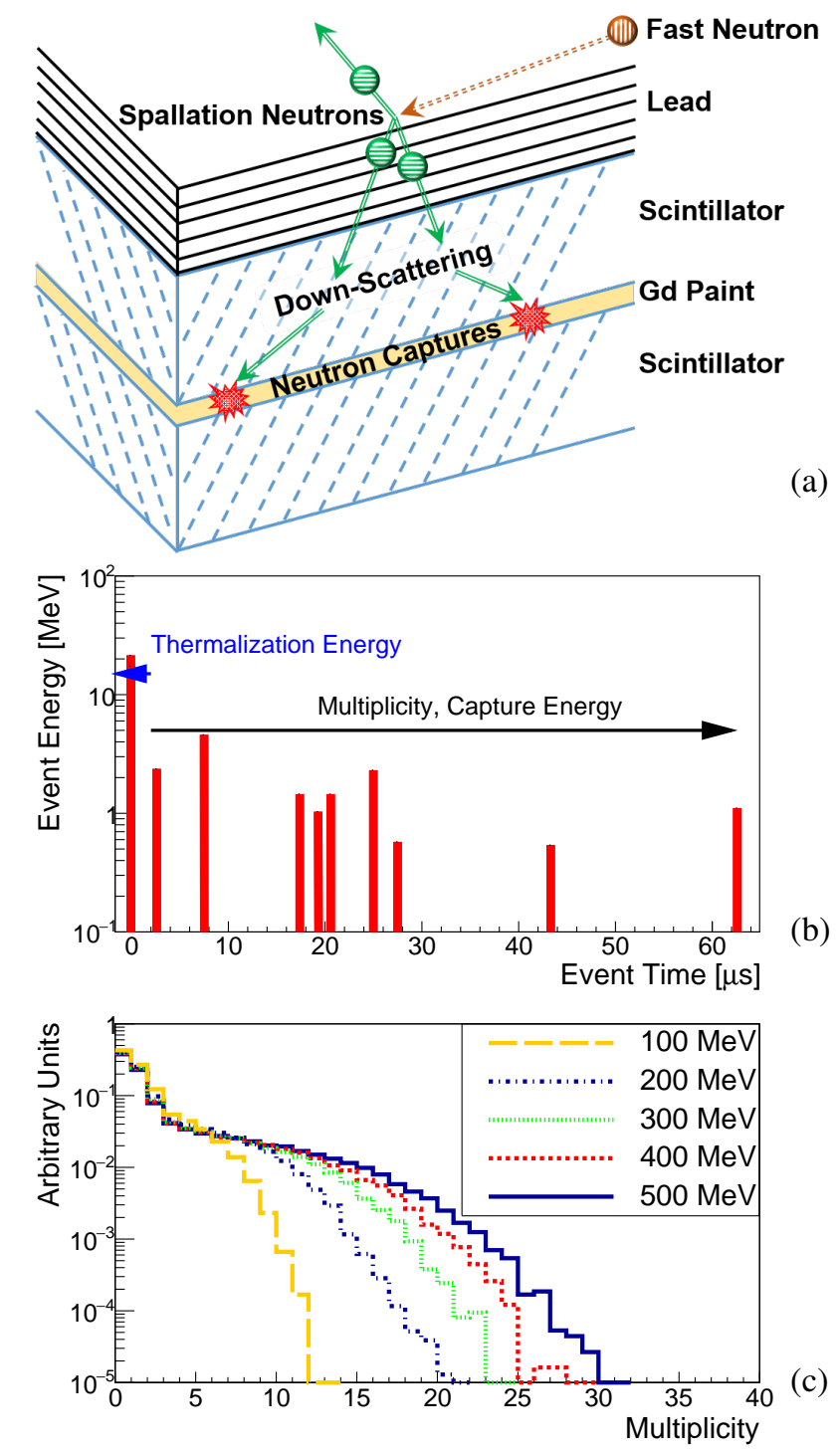

Figure 2: (a) Depicts a high-energy neutron initiating a spallation reaction in the lead and generating secondary neutrons. These secondary neutrons down-scatter in the scintillator and are captured on a Gd nucleus in the paint. The Gd de-excitation produces 1-5 gamma rays which further interact in the scintillator. (b) Depicts the timing characteristics of a high-energy neutron multiplicity event. An event is characterized by the number of neutron captures recorded (Multiplicity), the total energy deposited by the Gd de-excitations (Capture Energy), and the neutron energy deposited during down-scattering (Thermalization Energy). (c) Depicts the predicted secondary neutron multiplicity response for several different primary neutron energies in MARS. 


\subsection{Data Acquisition System}

The MARS DAQ is composed of four 14 bit, $250 \mathrm{MHz}$ Struck SIS3316 sixteen channel Waveform Digitizers (WFD) [10] and a CAEN 1495 General Purpose Board programmed to produce a $240 \mathrm{MHz}$ clock for board synchronization [11]. Each neutron detector is readout by one WFD, while the veto detectors are split between two WFDs.

Firmware programming in the SIS3316 WFD calculates and records accumulated charge values for 8 independently configurable time ranges. Full waveforms are not recorded to reduce data rates. To characterize the signal three of the time ranges are used by the current analysis: a $100 \mathrm{~ns}$ pre-pulse recorded before the trigger to measure the WFD pedestal, $300 \mathrm{~ns}$ after the trigger to integrate the pulse, and a final $300 \mathrm{~ns}$ time range beginning $300 \mathrm{~ns}$ after the trigger is used to indicate pulse pileup. All WFDs use group triggering: the signals of all sixteen PMTs in a neutron detector are recorded if a group of four PMTs exceeds a threshold. The veto group triggering is slightly different: the associated group of four PMTs is recorded only if that group passes the associated threshold. The top veto fishtail panels are recorded as 2 groups. Each overlapping pair of side veto fishtail panels belong to a group and each overlapping pair of square veto panels belong to a group. By using this group triggering scheme for the detection system, dark-noise triggering is reduced.

\section{Monte Carlo Model}

The MARS Monte Carlo package was designed to predict the detector response to gamma rays, neutrons, and muons. The following section describes the physics models chosen to simulate the response from these particles and provides a prediction of the muon veto efficiency.

\subsection{The Model}

Geant4.9.6.p02 [4, 5] was used with the Shielding physics list to model the detector response. A post-processing simulation analysis was used to appropriately quench particle interactions [12], apply the integration time ranges described in Sec. 2.4, and apply the position dependent response described in Sec. 4. Using these models and analysis two problems were encountered in regards to simulating the predicted neutron response: poor modeling of the multiplicity and total energy of Gd de-excitation gamma rays originally identified by Horton-Smith [13] and a poor reproduction of the inelastic reaction channels and kinematics for neutron-carbon interactions above $20 \mathrm{MeV}$ as shown first by Roeder [14].

The evaporation model was used to simulate Gd de-excitations. In contrast to the the default final state model, the evaporation model conserves energy and more accurately reproduced the gamma ray multiplicity for the Gd deexcitation. However the evaporation model underpredicts the 1-2\% of decays that result in a single gamma ray at an energy near the $\mathrm{Q}$ value.

The MENATE_R package [14, 15] was used to simulate inelastic neutron carbon interactions. MENATE_R considers six inelastic reaction channels: $\left(\mathrm{n}, \mathrm{n}^{\prime} 3 \alpha\right),(\mathrm{n}, \alpha),\left(\mathrm{n}, \mathrm{n}^{\prime} \mathrm{p}\right),(\mathrm{n}, \gamma),\left(\mathrm{n}, \mathrm{n}^{\prime} \mathrm{n}\right)$, and $(\mathrm{n}, \mathrm{p})$ which were tuned to results 
from several experiments [16, 17]. In contrast to several Geant4 cascade models, MENATE_R does not over-predict the $(\mathrm{n}, \gamma)$ reaction channel [14, 15].

Using the aforementioned physics models and the dimensions described in Sec. 2, the simulation includes the neutron detectors, the lead, the veto system, and the deployment platform as displayed in Fig. 3

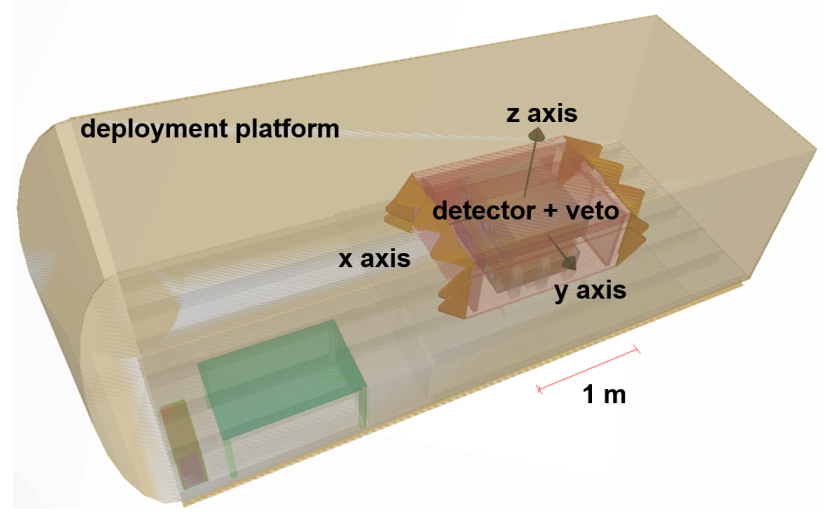

Figure 3: A depiction of the computer rendering of the MARS detector system inside the deployment platform. Simulation axes and the model scale are included.

\subsection{Muon Veto Efficiency}

Neutrons, muons, and a range of other cosmogenic high-energy particles induce spallation reactions on the lead target described in Sec. 2 If not identified by the veto system, muons can interact in the lead producing multiple neutrons, which appear similar to a high energy neutron event.

Using the previously described models the muon identification efficiency was investigated. In all deployment scenarios the non-identified muon rate could be problematic if it overwhelmed the desired neutron flux. In underground deployment scenarios muogenic neutrons are expected to be strongly forward peaked with the incident muon direction [18], making it difficult to disentangle the muon and neutron components. Due to the potentially entangled nature of the underground signal, the muon identification efficiency of MARS was modeled using a Monte Carlo simulation.

In above ground deployment scenarios, the energy and incident direction of muons are sampled using the CRY package for Geant4 [19]. Underground muons are sampled from the distribution described by Reyna [20] and propagated to the appropriate depth using a site specific overburden map. The Reyna distribution was used underground to slightly reduce computation time. Here the muon veto efficiency was characterized for an underground deployment scenario at KURF at a depth of 600 m.w.e.

Muons that deposited energy in the detector, but not in the veto were counted as unidentified muon events. No cuts were applied based upon the detected multiplicity. Detector and veto position dependent response calibrations described in Sec. 4 as well as software thresholds were applied to all energy depositions. Dividing the number of missed muon events by the number of muons passing through the detector or veto, a muon identification efficiency of $99.995 \pm 0.002 \%$ was predicted, where the error incorporates only statistical uncertainty. 


\section{Detector Calibrations and Characterization}

To ensure a time-independent response over a large dynamic range for the PMTS in MARS, two pre-processing corrections were made to the data: accounting for gain drift over time and the non-linear response of the PMTs to light. The following section describes the gain drift and linearity corrections. After the corrections, the energy calibration and position dependent response was determined using the Monte Carlo model described in Sec. 3 and the measurement of Cs-137 and Co-60 sources.

\subsection{Gain Drift}

To account for changes in the detector configuration that cause gain drift (PMT drift, mechanical coupling, etc.), a time-independent response can be achieved by gain matching the PMTs by the single photo-electron (PE) spectra before and during an experiment. However the gain of the 5 inch diameter PMTs was not sufficient to observe single PEs over the noise of the WFD and associated electronics. A rough estimate of the measured single PE response was obtained for a subset of the PMTs using an oscilloscope in place of the WFDs. This estimated PE response is used to compare gain drift in the following section.

In the absence of a single PE peak the muon energy distribution was found to be the most consistent feature throughout a measurement period. While the muon energy and angular distribution can change depending on the deployment scenario, muons passing perpendicularly through both neutron detectors and the lead deposit roughly the same energy corresponding to the peak at 10,000 PE in Fig. 4 a. The rate at which muons interact in only one neutron detector, depositing a significantly larger or smaller energy than perpendicular muons, changes the tails of the muon peak distribution in Fig. 4 a but does not change the peak position.

Muon events were identified by large energy depositions in the detector in coincidence with the surrounding veto. Each PMT's gain was adjusted based upon the position of the peak in the muon energy distribution relative to its position at the beginning of initial construction. The threshold for muon identification was applied to the detector energy (sum of all 16 PMTs) shown in Fig. 4 a to the right of the dotted vertical line. The detector count rate with a coincident muon veto event is displayed as a function of energy in Fig. $4 \mathrm{p}$ for a representative PMT from the neutron detector. The blue data with no marker is uncorrected data from the 1450 m.w.e. depth of KURF. The red data with a triangle marker was taken at KURF at the 600 m.w.e. depth. The 600 m.w.e. depth was selected as the reference gain template. Due to the increased rock overburden the uncorrected data from the 1450 m.w.e. depth had roughly an order of magnitude lower muon rate than the template data. A transformation described by Eq. 1 was applied to all points in the blue unmarked data to produce the corrected black square marker data:

$$
\begin{aligned}
& x \rightarrow G x, \\
& y \rightarrow a G y,
\end{aligned}
$$

where $x$ and $y$ before the transformation were the measurement in PE and rate respectively, and after the transform are the corrected measurement in PE and rate respectively, $a$ is the vertical overburden scaling factor, and $G$ is the PMT 
gain factor. A $\chi^{2}$ minimization with respect to $a$ and $G$ was performed using ROOT's MINUIT2 [21] package from $\sim 150$ PE to $\sim 1000$ PE to produce the correction. The $\chi^{2}$ function used was

$$
\chi^{2}=\sum_{i}\left(\frac{y_{c o r r, i}-y_{t e m p, i}}{\sigma_{c o r r, i}}\right)^{2},
$$

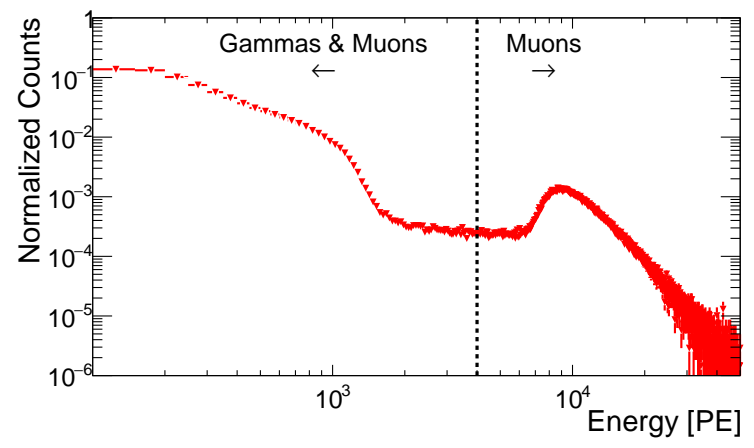

(a)
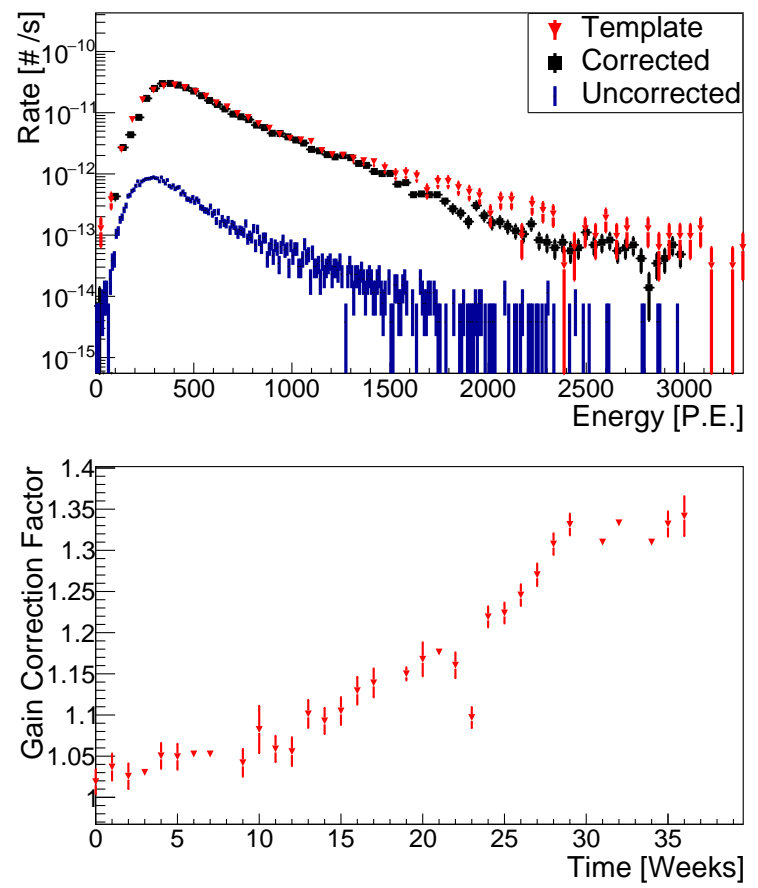

(c)

Figure 4: (a) The total neutron detector energy spectrum is shown. The edge at 1,000 PE is due to Gd de-excitations and the peak at 10,000 PE is due to through going muons. (b) A representative gain correction plot for a PMT in the neutron detector is shown. The blue data with no marker is the pre-corrected data, the red data with a triangular marker is the template data, and the black data with the square marker is the corrected data. (c) Displays the gain correction factor $G$ as a function of time in weeks for the same PMT (b). The gain degradation is assumed to be due to mechanical decoupling.

Gain matching the muon energy distribution was performed on a 7-14 day basis depending on the muon rate and 
it was assumed that the gain was relatively constant over this time span. An example time dependent gain correction is displayed in Fig. 4 for the same PMT as Fig. 4p. The detector and deployment platform were moved at week 23 resulting in the gain shift observed. Slow gain degradation was observed for a stationary detector over a many week period. The degradation was assumed to be the result of mechanical decoupling. At the end of the 37 weeks displayed in Fig. 4k, the average gain correction for the neutron detector PMTs was $1.26 \pm 0.12$.

\subsection{Non-Linearity}

Non-linear response in PMTs can occur when sufficient charge is introduced into the dynode structure to induce space-charge effects. In this situation, large numbers of electrons decrease the accelerating electric field limiting further electron generation on the dynodes [22]. To measure the PMT non-linearity, PMT calibrations were performed before the MARS detector was assembled. Various combinations of multiple light emitting diodes (LEDs) were used to measure the PMT response to a wide range of light levels, and to determine the relationship between these combinations and single LEDs at a fixed drive pulse voltage. For example, if the PMT response to a pair of LEDs pulsing is less than the sum of the PMT response to those LEDs pulsing separately, that would reveal a non-linearity in the PMT response.

Each PMT and three independently driven LEDs were placed horizontally in a light tight box. The LEDs were closely spaced together and independently connected to a fast square pulse generator. All combinations of the three LEDs were pulsed at a fixed LED voltage and the PMT response was recorded by the DAQ. That is, the response was recorded for each LED pulsing individually, each pair pulsing simultaneously, and all three pulsing simultaneously. All combinations were covered within a few minutes at a given drive pulse voltage to minimize the effect of the variation of LED light output with ambient temperature. Initially the LEDs were driven at low voltages so that only a small signal was observed; here the PMT was assumed to operate in a linear regime. The driving voltage was adjusted so that the range of light outputs spanned by the LED permutations at a given voltage overlapped with those at adjacent voltages. In this way the non-linear response curve of the PMT was measured via "boot-strapping" upwards from the few PE level. Results are plotted in Fig. 55 for a representative PMT. Linearity corrected values were found by spline interpolating Fig. 5 ] using ROOT's TGraph [21].

\subsection{Energy Calibration}

Using the gain drift and linearity corrections, four sets of measurements and simulations were performed to characterize the detector energy calibration and position dependent response. The detector energy calibration was used to transform the measured WFD value to energy and the position dependent response was used on subsequent simulations to approximate the detector light transport. All energy calibrations performed in this section utilized a Co-60 and a Cs-137 gamma ray source. For all measurements and simulations acceptable fits were found over the range of the Compton valley to the Compton Edge.

The following measurements were performed: 


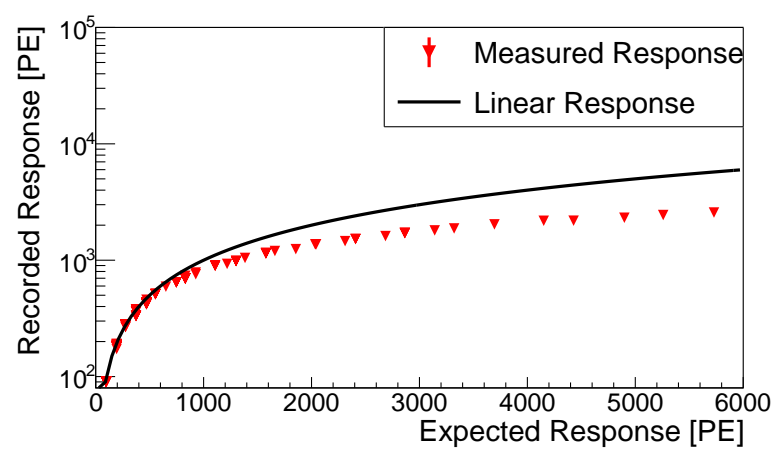

Figure 5: The measured PMT non-linear response as a function of light. The black line shows a perfectly linear response. The red triangular data points correspond to the PMT measured non-linearity. Errors for the red triangular data are too small to be observed over the marker.

1. The detector response in the center of a neutron detector

2. The position dependent response relative to the first measurement of a neutron detector

3. The position dependent response of the $72 \times 24 \times 1 \mathrm{~cm}^{3}$ veto paddles

4. The position dependent response of the $31 \times 28 \times 2 \mathrm{~cm}^{3}$ veto paddles

All measurements used the same algorithm to determine the energy calibration and detector response: a $\chi^{2}$ minimization was performed while linearly shifting the data and convolving the simulation with a Gamma distribution. To describe the linear shift and convolution four free parameters were used:

1. $m$ : the conversion from the WFD value to calibrated energy

2. $b$ : the constant shift for the calibrated energy

3. $c$ : the constant convolution parameter

4. $d$ : the energy dependent convolution parameter

The $\chi^{2}$ function used was

$$
\chi^{2}=\sum_{i}\left(\frac{E_{\text {exp }, i}-E_{\text {sim }, i}}{\sigma_{\text {exp }, i}}\right)^{2},
$$

where $E_{\text {exp }}$ is the shifted experimental spectrum, $E_{\text {sim }}$ is the convolved simulated spectrum, and $\sigma_{\text {exp }}$ is the shifted experimental spectrum error. The experimental spectrum was shifted by

$$
E_{\text {exp }}=m * W F D+b
$$

where $W F D$ is the value of the WFD pulse integral as described in Sec. 2. The simulation spectrum was convolved with a Gamma distribution

$$
E_{\text {sim }}=\int S(E) * G(E, k, \theta) d E
$$

where $S(E)$ is the energy dependent original simulation spectrum, $G(E, k, \theta)$ is the energy dependent Gamma distribution, $k$ is the Gamma shape parameter, and $\theta$ is the Gamma scale parameter. The Gamma distribution parameters 
were calculated using the fit parameters $c$ and $d$ :

$$
\begin{array}{r}
\sigma=c+d \sqrt{E}=\sqrt{k} \theta, \\
\mu=E=k \theta .
\end{array}
$$

The first measurement for the energy calibration was in the center of the top neutron detector using the above algorithm. A collimator with slit dimension $8 \times 0.4$ inch $^{2}$ was fashioned out of four $8 \times 4 \times 2$ inch $^{3}$ lead bricks to restrict the measured detector response to the center of the detector. The four lead bricks were placed with the $8 \times 4$ inch $^{2}$ face in contact with the detector. The collimator was placed at the center of the detector, with the 8 inch length running parallel to the detector $100 \mathrm{~cm}$ side, and $1 \mu \mathrm{Ci}$ of Cs-137 and Co-60 were separately measured. The convolved simulated energy and calibrated experimental spectrum are shown in Fig. 6. The Gamma distribution used in Eq. 5 was found to be a poor model of the energy resolution observed in regions outside the Compton edge. A more robust model to fully describe the light collection efficiency controlling the energy resolution is not considered feasible given the complicated internal geometry of the scintillator sheets, acrylic, and associated air-grease boundaries.

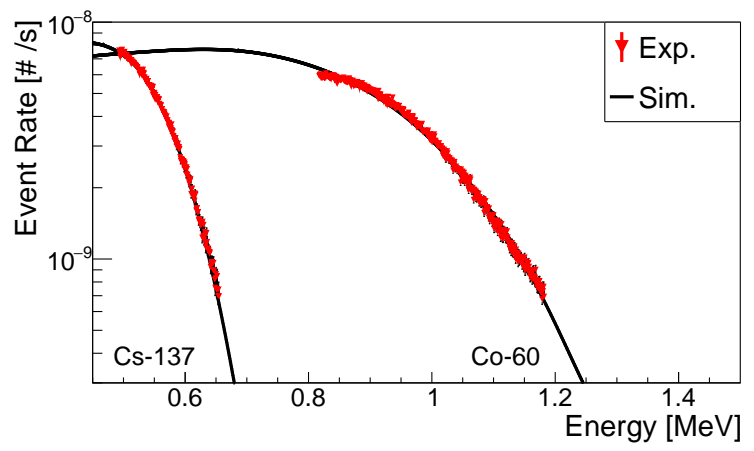

Figure 6: The experimental and simulated Cs-137 and Co-60 spectra in the center of the detector with the collimator are shown from left to right respectively. The experimental data is red with a triangle marker and the simulation data is a black solid line.

The second measurement accounted for the position dependent response relative to the center calibration of the neutron detector. Spectra from Co- 60 were measured in a $5 \times 5$ grid on the top neutron detector. Only Co- 60 was used due to a prohibitively long measurement time for the Cs-137 source that was available. Due to the position dependent calibration only having one source, the number of fit parameters were reduced by fixing the center calibration values and adding one multiplicative factor $T$ :

$$
\begin{aligned}
E_{\text {exp }} & =m_{f} * T * W F D+b_{f}, \\
\sigma & =c_{f}+d_{f} \sqrt{T * E}=\sqrt{k} \theta,
\end{aligned}
$$

where all parameters with the subscript $f$ denote previously defined parameters from Eqs. 4 and 6 . The subscript $f$ parameters were fixed to the same value as the calibration in the center of the top neutron detector. The only free fit parameter was $T$. 
The third measurement used uncollimated Cs-137 and Co-60 sources separately at the center and both edges of the $72 \times 24 \times 1$ inch $^{3}$ veto paddles. The last measurement used the same uncollimated sources at the center and one corner of the $31 \times 28 \times 2$ inch $^{3}$ paddles. Both the third and fourth measurements used the $\chi^{2}$ analysis described by Eqs. 36

\section{Multiplicity Event Identification}

After the calibrations and characterizations described in Sec. 4, different multiplicity identification algorithms were investigated. In experiments without a triggered start time, fast neutron multiplicity events are difficult to detect: no consistent signal exists to identify the start of an event. Therefore, the multiplicity analysis identifies correlated energy depositions that form an event. The following section describes an algorithm for detecting correlated secondary neutron depositions given the known neutron capture time and the location dependent uncorrelated ambient gamma ray and neutron rate. No consideration is given to the primary neutron.

\subsection{Ranking Identification Algorithms}

A Monte Carlo model based upon the measured neutron capture time of $18.7 \pm 3.0 \mu \mathrm{s}$ (Sec. 6) was created to test various multiplicity identification algorithms. Additionally, the model included environmental and source induced background depositions which could be characterized by the distribution of time between energy depositions in the detector. If the background is uncorrelated, the distribution of time between depositions is a Poisson distribution. The triggering model assumed that the background depositions were uncorrelated and used an exponential fit of the time between depositions distribution to determine the characteristic time constant. In the rest of this paper the fitted characteristic time constant is referred to as the inter-event time.

To analyze different triggering algorithms, the neutron capture time was sampled to simulate $n$ neutron captures starting from time zero. The uncorrelated gamma ray and neutron background was included by sampling the background distribution, starting two inter-event times before the first neutron capture until two inter-event times after the last neutron capture. One million sequences of this nature were simulated with $n$ ranging from 3 to 10 .

For measurements where the neutron flux is expected to be significantly larger than the background flux, the effectiveness of different triggering algorithms was compared by examining the neutron detection efficiency and the number of background depositions identified as correlated neutrons. A figure of merit $\left(F O M_{\text {Trig }}\right)$ was defined:

$$
\operatorname{FOM}_{\text {Trig }}(n)=\frac{m / n}{b+1}
$$

where $n$ is the number of true neutron captures, $m$ is the number of detected neutron captures, and $b$ is the number of background depositions identified as neutron captures within a multiplicity event. Each triggering algorithm was used to process the depositions into events and calculate the figure of merit $\left(F O M_{\text {Trig }}\right)$, which is shown in Fig. 77a. Due to the $F O M_{\text {Trig }} \propto \frac{1}{n}$ the optimal value will always be less than 1 . 
For measurements where the neutron flux is not known, or less than or on the order of the background flux, the $F O M_{\text {Trig }}$ is insufficient to rank trigger algorithms. In addition to the $F O M_{\text {Trig }}$, the rate at which events composed of only background depositions were misidentified as multiplicity events was used to characterize the triggering algorithms. The background multiplicity rate was determined by sampling the inter-event time distribution. The same triggering algorithms were used to detect background multiplicity events masquerading as correlated secondary neutrons. The resulting background multiplicity spectra was normalized to the time simulated and shown in Fig. 7p.

An optimal triggering algorithm has a $F O M_{\text {Trig }}$ close to 1 with the comparatively smallest background multiplicity rate. In deployment scenarios where the background rate and a reliable estimate of the fast neutron flux is known further optimization may be possible.

\subsubsection{Identification Algorithms}

Two types of multiplicity identification algorithms were investigated: a fixed length time range and an expanding length time range. Both algorithms defined an initial time range, started the time range at the time of the first available deposition, and required three or more depositions to record an event. If three or more depositions were not recorded in the time range the first deposition was discarded and the time range was updated to start at the second deposition. If the three deposition threshold was reached and an event was ended the next available deposition started the next event. The $F_{O M}$ Trig and background multiplicity rate for several fixed and expanding time range identification algorithms are shown in Fig.7.

Fixed time ranges from 25 to $200 \mu$ s were investigated. A fixed time of $65 \mu$ s was found to be optimal for measurements with a strong neutron flux compared to the background. For the expanding time range algorithm, if 3 or more depositions were reached the gate was extended by a secondary time range from the time of the second to last deposition in the event. Using this algorithm the initial time range was shorter than the fixed length algorithm, which rejected background depositions more efficiently at the cost of detection efficiency for low neutron multiplicities. The expanding nature of the time range allowed the algorithm to detect high multiplicity events more efficiently than the fixed gate algorithm as shown in Fig. 7 a. Initial time ranges of 25 to $100 \mu$ s and secondary time ranges from 25 to $100 \mu$ s were investigated. An optimal algorithm will depend on the expected background rate and the minimum multiplicity of interest.

\section{Neutron Capture Efficiency and Capture Time}

A Cf-252 source was used, given the experimental setup described below, to determine the neutron capture efficiency and capture time of MARS. The comparison of the simulation and experimental data given this setup allows for the determination of the Gd loading in the Monte Carlo model. An appropriate Gd loading provides the best approximation of the secondary neutron capture efficiency and capture time regardless of the experimental setup. Using the information from Sec. 5 a multiplicity triggering algorithm of $65 \mu$ s was chosen for two reasons: the source had a higher flux than the uncorrelated gamma ray and neutron background, and the identification algorithm had the highest 

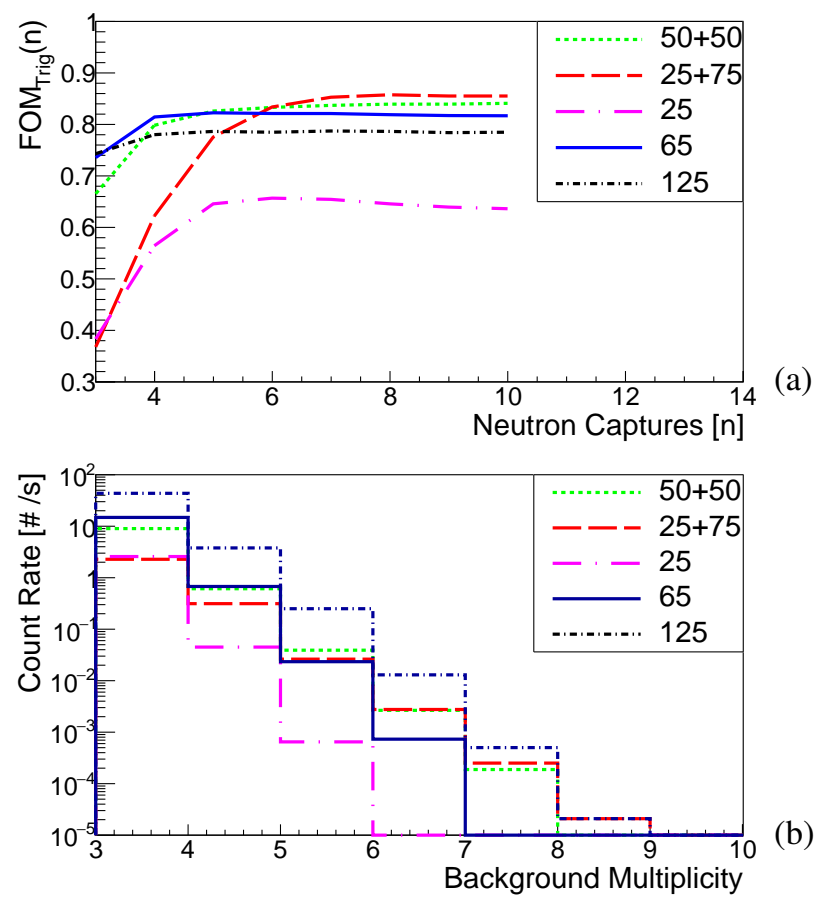

Figure 7: (a) The FOM Trig described in Eq. 8 for a variety of triggering algorithms described in Sec. 5.1.1 (b) The expected pure singles background multiplicity rate. Fixed length time ranges of 25, 65, and $125 \mu$ s are shown. Expanding time ranges are denoted with the initial range before and the secondary range after a + symbol.

FOM $M_{\text {rig }}$ for multiplicity 3-5 events. Multiplicity 3-5 events are important for the Cf-252 analysis because the mean neutron multiplicity of $\mathrm{Cf}-252$ is $3.757 \pm 0.01$ [23].

A Cf-252 source was positioned on top of four lead bricks above the top neutron detector. Lead bricks were used to reduce the number of prompt fission gamma rays interacting in MARS. In addition to the multiplicity event identification algorithm, a small $2 \times 2$ inch $^{2}$ cylindrical plastic scintillator detector coupled to a 2 inch PMT was used as a tagging detector to detect prompt fission gamma rays and neutrons. The tagging detector was situated as close to the Cf-252 source as possible. Multiplicity events were recorded if the first deposition started within $75 \mu$ s of a coincident deposition in the tagging detector; a $75 \mu$ s time range was necessary to ensure nearly uniform detection efficiency regardless of the true multiplicity. Any depositions in the multiplicity event within $100 \mathrm{~ns}$ of a coincident deposition in the tagging detector were discarded to remove the prompt fission gamma ray response. All measured parameters are a function of the solid angle of the source and detector and shielding provided by the lead. Due to this dependence the complete system is modeled in the simulation.

The Cf-252 multiplicity distribution was determined by:

$$
M(n)_{\text {meas }}=S\left(\sum_{i=n}^{N_{\max }} \beta(i) \epsilon^{n}+\sum_{j=0}^{j<n} \sum_{i=0}^{N_{\max }} \beta(i) \epsilon^{j} P_{b k g}(n-j)\right),
$$

where $n$ is the detected multiplicity number, $M(n)_{\text {meas }}$ is the measured multiplicity distribution, $S$ is the source 
strength, $N_{\max }$ is the maximum multiplicity considered (10 neutrons), $\beta(i)$ is the Cf-252 known neutron multiplicity distribution [23], $\epsilon$ is the neutron detection total efficiency, and $P_{b k g}$ is the Poisson probability for background events. The source strength $S$ and the total efficiency $\epsilon$ are unknown. The source background $P_{b k g}$ is modeled using the measured inter-event time of $478 \mu$ s and $\beta(i)$ is modeled using the parameters in Holden [23]. To eliminate the unknown source term $S$ a ratio between different bins of the measured multiplicity spectrum $M(n)_{\text {meas }}$ can be used. The resulting ratios are only dependent upon the efficiency $\epsilon$. These equations can be solved graphically. Using the following ratios: $\mathrm{M}(3) / \mathrm{M}(4), \mathrm{M}(3) / \mathrm{M}(5)$, and $\mathrm{M}(4) / \mathrm{M}(5)$ the total efficiency $\epsilon$ was calculated to be $12.8 \pm 0.5 \%$ and $12.7 \pm 0.3 \%$ for the experimental data and simulation respectively. The quoted error for the total efficiency $\epsilon$ only includes statistical error. The results for each multiplicity distribution ratio are displayed in Table 1 . Agreement

Table 1: Various multiplicity ratios were used to calculate the total neutron detection efficiency measured with a Cf-252 source.

\begin{tabular}{ccc}
\hline \hline Ratio & Exp. $\epsilon(\%)$ & Sim. $\epsilon(\%)$ \\
\hline $\mathrm{M}(3) / \mathrm{M}(4)$ & $13.0 \pm 0.4$ & $12.8 \pm 0.2$ \\
$\mathrm{M}(3) / \mathrm{M}(5)$ & $12.8 \pm 0.6$ & $12.7 \pm 0.4$ \\
$\mathrm{M}(4) / \mathrm{M}(5)$ & $12.6 \pm 1.4$ & $12.6 \pm 0.8$ \\
\hline Average & $12.8 \pm 0.5$ & $12.7 \pm 0.3$ \\
\hline \hline
\end{tabular}

between experimental data and simulation was observed for all ratios within $1 \sigma$.

Using the same experimental data and simulation the capture time distribution was calculated by

$$
\begin{aligned}
P_{\text {capture }}(t) & =P_{\text {meas }}(t)- \\
& \sum_{n=3}^{n<N_{\max }} \sum_{j=0}^{j<n} \sum_{i=0}^{N_{\max }} \beta(i) \epsilon^{j} P_{b k g}(n-j, t),
\end{aligned}
$$

where $P_{\text {capture }}(t)$ and $P_{\text {meas }}(t)$ are the background corrected and measured capture time distributions respectively, and all other parameters have previously been defined in Eq. 9 The capture distributions $P_{\text {capture }}(t)$ and $P_{\text {meas }}(t)$ are constructed using the individual deposition time after the tagging detector. For example a multiplicity 3 event would have 3 entries in $P_{\text {meas }}(t)$. The experimental and simulated capture times were $18.7 \pm 3.0 \mu \mathrm{s}$ and $20.0 \pm 0.1 \mu \mathrm{s}$ respectively and the distributions are shown in Fig 8 . The relatively small error on the simulated capture time only incorporated statistical uncertainty.

\section{Fast Neutron Effective Area Predictions}

The validated Monte Carlo model was used to predict the effective area for incident high-energy neutrons in MARS. Using the algorithm described in Sec. 5with an inter-event time of $\sim 680 \mu$ s corresponding to the uncorrelated gamma ray and neutron background rate at the 600 m.w.e. depth at KURF, the optimal trigger was found to be an 


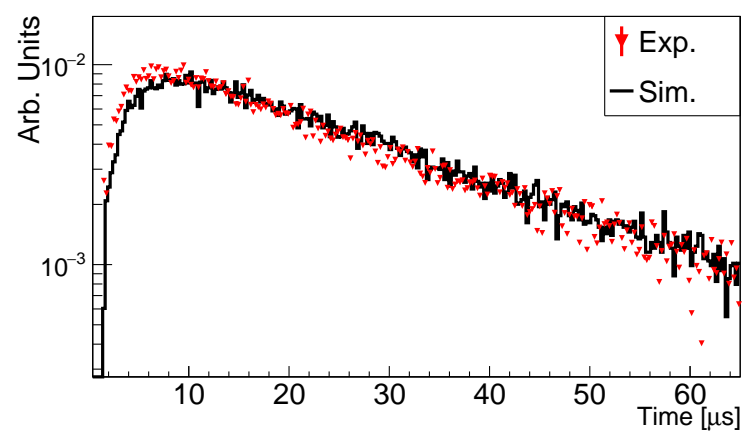

Figure 8: Capture time distribution for the experimental data and simulation from tagged multiplicity events from a Cf-252 neutron multiplicity source. Some disagreement exists at short capture times but the exponential shape matches reasonably well.

expanding length time range with an initial time of $25 \mu$ s and an expansion of $75 \mu \mathrm{s}$. This expanding trigger was used due to its large $F O M_{\text {Trig }}$ for higher multiplicities and its relative small background multiplicity rate.

Fast neutrons of 25 to $1000 \mathrm{MeV}$ were simulated using the MARS Monte Carlo model described in Sec. 3 without the deployment platform. Primary neutrons were generated on a half sphere of radius $1.9 \mathrm{~m}$ surrounding the detector. The recorded energy depositions for all active detector and veto volumes were grouped by the time ranges described in Sec. 2.4 to determine the energy, weighted position, and time. Energy and position dependent responses outlined in Sec. 4 were used to convolve the simulated response. The expanding time range triggering algorithm described in Sec.5.1 was used to determine the multiplicity, capture energy, and thermalization energy. Three additional thresholds were set in decreasing order of data rejection:

1. Each deposition had greater than $0.8 \mathrm{MeV}$ deposited energy.

2. The multiplicity was greater than or equal to 5

3. The capture energy was greater than $10 \mathrm{MeV}$

An energy threshold of $0.8 \mathrm{MeV}$ was chosen to be safely above the gain varying hardware threshold. A multiplicity threshold of 5 was chosen for two reasons: the detector response difference observed above 5 in Fig. 2 r and to reduce the uncorrelated gamma ray and neutron backgrounds in the experimental data. A capture energy threshold of $10 \mathrm{MeV}$ was chosen based upon the expected uncorrelated gamma ray background response for multiplicity 5 events.

In addition to the multiplicity mode, a capture-gating analysis was performed. Using both modes ensured the evaluation of the detector performance over a larger range of energies and facilitated a trade-off study between the two modes. For the capture-gating mode two thresholds were set in decreasing order of data rejection:

1. The first deposition must deposit more than $10 \mathrm{MeV}$

2. The down-scatter and capture must occur within $100 \mu \mathrm{s}$

The first deposition threshold was set above the Gd de-excitation energy of $8 \mathrm{MeV}$ so that the deposition was identified as a neutron scatter. The $100 \mu$ s time range was chosen to ensure a high detection probability for neutron captures 
and the subsequent Gd de-excitation. Based upon the measured $680 \mu$ s inter-event time, background depositions in the $100 \mu$ s time range would be at an acceptable level.

The effective area for both detection modes for incident neutrons with isotropic, $\cos ^{2}(\theta)$, and $\cos ^{3}(\theta)$ angular distributions is shown in Fig. 9. The effective area was calculated by

$$
A_{\text {eff }}\left[\mathrm{cm}^{2}\right](E)=\frac{N_{\text {interacting }}(E) *(2 \pi * 190 * 190)\left[\mathrm{cm}^{2}\right]}{N_{\text {simulated }}(E)},
$$

where $A_{\text {eff }}\left[\mathrm{cm}^{2}\right](E)$ is the effective area, $N_{\text {interacting }}(E)$ is the number of neutrons events passing the respective threshold, $(2 \pi * 190 * 190)\left[\mathrm{cm}^{2}\right]$ is the surface area of the generating surface, and $N_{\text {simulated }}(E)$ is the total number of neutrons simulated. A more generic description of calculating the effective area is described by Sullivan [24]. The underground neutron angular distribution is expected to be an energy dependent combination of isotropic and highly peaked along the initiating muon direction [25]. Incident neutrons which follow the initial muon angular distribution are likely to be removed from the recorded data set due to a coincidence with the muon veto. Above ground the angular distribution is expected to be at least $\cos ^{2}(\theta)$ and most likely $\cos ^{3}(\theta)$ [26]. The multiplicity mode effective area is plotted in black with different line styles for each distribution. The capture-gating mode effective area is plotted in red with same line styles as the multiplicity mode for each respective distribution.

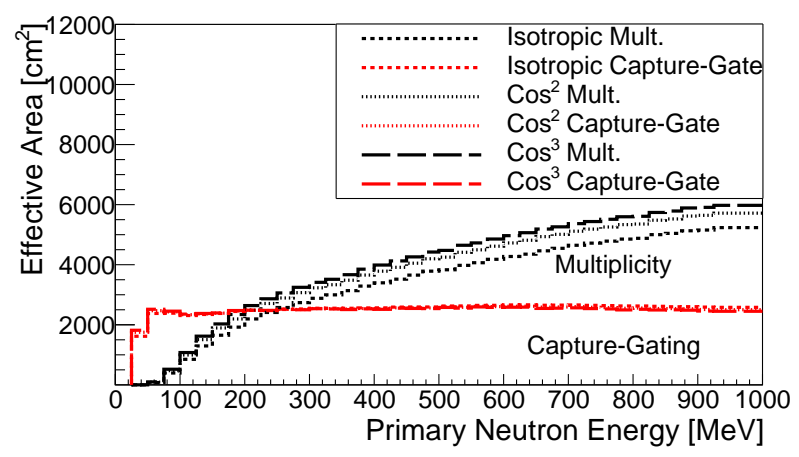

Figure 9: The predicted effective area of isotropic fast neutrons in the MARS detector using the multiplicity and capture-gating modes. Increasing effective area is observed for neutron energies above $50 \mathrm{MeV}$ with the multiplicity mode. The efficiency is observed to flatten as a function of energy for the capture-gating mode above $100 \mathrm{MeV}$. The bin width is $25 \mathrm{MeV}$.

Examining Fig. 9, the effective area of MARS for capture-gating and the multiplicity mode intersect at different energies depending on the simulated angular distribution. Figure 9 should not be used to generalize that the multiplicity technique is superior to the capture-gated technique above $200 \mathrm{MeV}$. It is intended to display trends in the effective area between the different analyses in a fixed detector. With this in mind, the effective area did not account for background rejection and event identification characteristics. The multiplicity mode may have a higher signal-to-noise ratio for incident high-energy neutrons due to requirement that many secondary neutrons be detected, particularly above $100 \mathrm{MeV}$. These multiplicity events are comparatively easier to distinguish from single neutron or gamma ray backgrounds than the expected signal from a capture-gating event due to the number of correlated depositions. Due 
to the these characteristics, the MARS analysis uses the multiplicity mode above $100 \mathrm{MeV}$. Future analyses which account for the different signal to background characteristics of each mode may provide a cross-check of both techniques in a small but overlapping energy regime. If both techniques agree, it may be possible to increase the detection efficiency of MARS by combining both data sets.

The most statistically challenged measurement location currently investigated by MARS is the 1450 m.w.e. depth at KURF. While no measurements of the flux have previously been made at a depth near 1450 m.w.e., the energy dependent flux has been predicted by Mei and Hime at depth of 1585 m.w.e using Monte Carlo simulation [25]. The 1585 m.w.e depth is the most shallow depth considered by Mei and Hime. The predicted event rate at the 1450 m.w.e. depth at KURF can be approximated by using the effective area for the multiplicity mode from Fig. 9 and the WIPP curve from Fig. 17 from Mei and Hime [25]. This event rate is a crude approximation that ignores overburden and rock composition differences. Using this expected event rate and the known live-time at the 1450 m.w.e. depth, MARS has the potential to detect sufficient events to reconstruct a coarsely binned energy dependent flux above $100 \mathrm{MeV}$.

\section{Conclusions}

MARS is a transportable fast neutron detection system designed to measure neutron spectra and flux ranging from tens to hundreds of MeV. In contrast to previous approaches, MARS uses two detection modes: a conventional capture-gating mode for measuring neutron energies and a novel multiplicity mode for measuring neutron energies above $100 \mathrm{MeV}$. These detection modes increase the sensitivity of the detector and allow for the measurement of neutron fluxes of lower intensity than previous approaches.

MARS was constructed using plastic scintillator interleaved with Gd painted Mylar sheets, arranged around a lead table. Primary neutrons from a capture-gated event or secondary neutrons produced in the lead from a multiplicity event down-scatter, thermalize in the scintillator, and are captured on a Gd nucleus. The Gd nucleus de-excitation produces 1-5 gamma rays of total energy $\sim 8 \mathrm{MeV}$ which interact in the plastic scintillator.

A Geant4 Monte Carlo model of the detector, muon veto, and deployment platform was constructed. Monte Carlo simulations and experimental data were used to validate the detector response to gamma ray and fission neutron sources. The validated Monte Carlo model was used to predict the effective area for the multiplicity and capture-gated modes. For incident neutron energies between $100 \mathrm{MeV}$ and $1000 \mathrm{MeV}$ with an isotropic angular distribution, the multiplicity analysis predicted an effective area of $500 \mathrm{~cm}^{2}$ rising to $5000 \mathrm{~cm}^{2}$. For neutron energies above $20 \mathrm{MeV}$, the capture-gating analysis predicted an effective area between $1800 \mathrm{~cm}^{2}$ and $2500 \mathrm{~cm}^{2}$. The effective area for the multiplicity mode was found to increase with the power $\cos (\theta)$ distribution. The capture-gating mode was found to be insensitive to the simulated angular distributions.

The first fast neutron measurements by MARS was performed at KURF. Data analysis is currently ongoing for the three at depth measurements $(380,600$, and 1450 m.w.e.). Of the three measurements, the 1450 m.w.e. depth is most statistically limited. At this depth, Monte Carlo simulations using the predicted neutron flux distribution, the predicted 
effective area, and the measurement live-time indicate that a sufficient number of events may have been measured to permit spectral unfolding.

As a verification of a well characterized source, above ground measurements are currently underway. Future work will encompass choosing, optimizing, and using an unfolding algorithm, with special emphasis on error propagation.

\section{Acknowledgments}

This material is based upon work supported by the Department of Energy National Nuclear Security Administration under Award Number: DE-NA0000979 through the Nuclear Science and Security Consortium.

This work was performed under the auspices of the U.S. Department of Energy by Lawrence Livermore National Laboratory under Contract DE-AC5-07NA27344. LLNL-JRNL-677776.

Sandia National Laboratories is a multi-program laboratory managed and operated by Sandia Corporation, a wholly owned subsidiary of Lockheed Martin Corporation, for the U.S. Department of Energys National Nuclear Security Administration under contract DE-AC04-94AL85000. Not approved for unlimited release, SAND2015365427.

We would like to thank J. Brennan for his work in constructing the MARS detector. We would like to thank J. Steele for his work on the DAQ.

\section{References}

[1] L. Garrison, Measurement of Neutron and Muon Fluxes $100 \mathrm{~m}$ Underground with the SciBath Detector, Ph.D. thesis, Indiana U., Bloomington (main) (2014).

[2] A. Malgin, O. Ryazhskaya, V. Ryasnyi, F. Khalchukov, High-Energy Hadrons Produced by Cosmic Ray Muons in the Earth as a Source of Background in Proton Decay Experiments, JETP Lett. 36 (1982) 376-379.

[3] A. Malgin, O. Ryazhskaya, Neutrons from muons underground, Physics of Atomic Nuclei 71 (10) (2008) 1769-1781.

[4] S. Agostinelli, et al., Geant4 a Simulation Toolkit, Nucl. Instr. and Meth. A 506 (3) (2003) $250-303$.

[5] J. Allison, et al., Geant4 developments and applications , IEEE Trans. Nucl. Sci. 53 (1) (2006) 270-278.

[6] A. Bernstein, et al., Nuclear Security Applications of Antineutrino Detectors: Current Capabilities and Future Prospects, Sci. Global Secur. 18 (2010) 127-192.

[7] R. Hennings-Yeomans, D. Akerib, A Neutron Multiplicity Meter for Deep Underground Muon-Induced High Energy Neutron Measurements, Nucl. Instr. Meth. A574 (2007) 89-97.

[8] M. Sweany, Characterization of Neutron Backgrounds for Direct Dark Matter Searches, Ph.D. thesis, University of California at Davis (2011).

[9] B133d01 photomultiplier tube, http://www .aditpmt.com/pmt-datasheets/B133D01W.pdf accessed: 2015-06-30.

[10] SIS3316 16 Channel VME Digitizer Family, http://www.struck.de/sis3316.html accessed: 2015-06-30.

[11] Technical Information Manual MOD. V1495 General Purpose VME Board, http://www.caen.it/servlet/checkCaenManualFile? Id=11043, accessed: 2015-09-30.

[12] Z. Jian-Fu, et al., Measurements of the Light Output Functions of Plastic Scintillator Using 9 Be(d, n) 10 B Reaction Neutron Source , Chinese Physics C 34 (7) (2010) 988. 
[13] G. Horton-Smith, Additional Gadolinium Support for GLG4sim, http://neutrino.phys.ksu.edu/ GLG4sim/Gd.html. accessed: 2015-07-08.

[14] B. Roeder, Development and Validation of Neutron Detection Simulations for EURISOL, EURISOL Design Study 3 (0) (2008) 31 - 44.

[15] P. Désesquelles, et al., Cross Talk and Diaphony in Neutron Detectors, Nucl. Inst. and Meth. A 307 (23) (1991) 366 - 373.

[16] R. Cecil, B. Anderson, R. Madey, Improved Predections of Neutron Detection Efficiency for Hydrocarbon Scintillators from 1 MeV to About $300 \mathrm{MeV}$, Nucl. Instr. and Meth. 161 (3) (1979) $439-447$.

[17] A. D. Guerra, A Compilation of n-p and n-C Cross Sections and Their Use in a Monte Carlo Program to Calculate the Neutron Detection Efficiency in Plastic Scintillator in the Energy Range 1-300 MeV , Nucl. Instr. and Meth. 135 (2) (1976) 337 - 352.

[18] V. Chazal, et al., Investigations of Fast Neutron Production by 190 !GeV/c Muon Interactions on Graphite Target, Nucl. Instr. Meth. A490 (2002) 334-343.

[19] C. Hagmann, D. Lange, D. Wright, Monte Carlo Simulation of Proton-Induced Cosmic-ray Cascades in the Atmosphere, http: //nuclear . 1lnl.gov/simulation/doc_cry_v1.7/cry_physics.pdf accessed: 2015-07-08.

[20] D. Reyna, A Simple Parameterization of the Cosmic-Ray Muon Momentum Spectra at the Surface as a Function of Zenith Angle arXiv: hep-ph/0604145

[21] R. Brun, F. Rademakers, ROOT - An Object Oriented Data Analysis Framework, Nucl. Instr. Meth. A389 (1997) 81-86.

[22] Photomultiplier tubes basics and applications, https://www.hamamatsu.com/resources/pdf/etd/PMT_handbook_v3aE.pdf accessed: 2016-02-29.

[23] N. E. Holden, M. S. Zucker, Prompt Neutron Multiplicities for the Transplutonium Nuclides , Radiation Effects 96 (1-4) (1986) $289-292$.

[24] J. Sullivan, Geometric factor and directional response of single and multi-element particle telescopes, NIM 95 (1) (1971) 5 - 11.

[25] D. Mei, A. Hime, Muon-Induced Background Study for Underground Laboratories, Phys. Rev. D73 (2006) 053004.

[26] J. Ziegler, Terrestrial Cosmic Rays , IBM Journal of Research and Development 40 (1) (1996) 19-39. 